\title{
Anke Lüdeling
}

\section{Einleitung zum Themenheft Empirie und Modellierung}

Prof. Dr. Anke Lüdeling: Institut für deutsche Sprache und Linguistik, Humboldt-Universität zu Berlin, Unter den Linden 6, D-10099 Berlin, E-Mail: anke.luedeling@hu-berlin.de

Unser diesjähriges Themenheft beschäftigt sich mit der Modellierung als einer Methode, die experimentelle Arbeit in der Linguistik unterstützen und weiterbringen kann. In den letzten Jahrzehnten sind in der Sprachwissenschaft viele empirische Methoden etabliert oder ausgebaut worden. Daten werden heute oft gezielter erhoben und transparenter zur Verfügung gestellt, Interpretationen besser abgesichert, Ergebnisse eher reproduzierbar. Die Fragestellungen für empirische Arbeiten kommen oft aus theoretischen Modellen und die Ergebnisse guter empirischer Arbeit wirken auf Theorien zurück. In vielen Theorien können Aussagen so formuliert werden, dass sie (jedenfalls im Prinzip) experimentell testbar sind.

Die experimentelle Absicherung ist natürlich für viele linguistische Teilgebiete wie die Soziolinguistik, die Sprachwandelforschung, die Spracherwerbsforschung oder Gebiete, die sich mit der menschlichen Sprachverarbeitung befassen, fundamental, aber auch in Gebieten, die traditionell eher nicht experimentell gearbeitet haben wie der Grammatikforschung, setzen sich experimentelle Methoden durch. Mit Experimenten können dabei Erhebungen, psycholinguistische oder neurolinguistische Studien genauso gemeint sein wie Korpusstudien (siehe Labov 2001, 2008, Manning 2003, Gilquin \& Gries 2009, Wulff \& Gries 2011, Ford \& Bresnan, erscheint und viele, viele andere). In vielen experimentellen Ansätzen geht es darum, Korrelationen zwischen kovariierenden Variablen zu etablieren oder Muster in (meist hochdimensionalen Räumen) zu finden. Dabei müssen statistische Modelle angewendet werden, da es oft nicht möglich ist, alle Daten, die man bräuchte, wirklich zu erheben, alle Zusammenhänge zu verstehen oder alle Abhängigkeiten vorherzusehen.

Die drei Artikel in diesem Themenheft beschäftigen sich daher mit der Frage, wie experimentelle Methoden und Modellierung zusammenwirken, um linguistische Fragestellungen neu zu adressieren oder ganz neue Fragen zu stellen. Alle drei Artikel sind dabei so konzipiert, dass sie nicht allein eigene neue Experimente vorstellen, sondern dass sie vielmehr die Möglichkeiten und Grenzen der jeweiligen Methode mitreflektieren. Sie zeigen, welche Fragestellungen mit der 
vorgestellten Methode beantwortet werden können, aber auch, für welche Fragestellung sie nicht geeignet ist.

Der Artikel ,Statistische Modellierung، von Stefan Thomas Gries erläutert einige Möglichkeiten der Modellierung von Sprachdaten mit statistischen Modellen. Er exemplifiziert die Modelle anhand von Korpusdaten und psycholinguistischen Daten. Die Modelle, die hier besprochen werden, versuchen, die Kovarianz zwischen sprachlichen Daten und anderen Daten (sprachlichen und außersprachlichen) zu ermitteln. Dabei ist Gries - neben vielen anderen Aspekten - wichtig, dass neben einzelnen kovariierenden Variablen unbedingt auch deren Interaktion betrachtet werden muss.

Die beiden anderen Artikel hängen sehr eng zusammen. Heiner Drenhaus und Peter beim Graben erklären in ,Ereigniskorrelierte Potenziale‘ die Möglichkeiten, die die Elektroenzephalographie bietet, menschliche Sprachverarbeitung (indirekt) dadurch zu beobachten, dass man neuronale Effekte bei bestimmten sprachlichen Aufgaben misst. Sie zeigen, dass frühere Untersuchungen, die bestimmte Effekte direkt auf syntaktische oder semantische ,Module‘ zurückgeführt haben, so nicht haltbar sind. Dies hat Auswirkungen auf die Theorie menschlicher Sprachverarbeitung - sowohl für Theorien über Modularität als auch für Theorien über Frequenz- und Erfahrungseffekte. Drenhaus und beim Graben erläutern die Messung ereigniskorrelierter Potenziale (EKP) anhand von Daten aus mehreren Studien zur Verarbeitung negativer Polaritätselemente. Diese Daten werden dann auch in dem Artikel ,Computationelle Neurolinguistik von beim Graben und Drenhaus betrachtet. In der computationellen Neurolinguistik sollen neuronale Effekte bei der Sprachverarbeitung modelliert werden. Dabei braucht man einerseits linguistische Modelle, die klare Vorhersagen über Akzeptanz bestimmter Daten machen und andererseits Modelle, die neuronale Änderungen bei der Verarbeitung dieser Stimuli vorhersagen. Beim Graben und Drenhaus bauen bei ihrem Modell auf eine Modifikation des Minimalismus und versuchen, das Verhalten der Versuchspersonen bei den realen EKP-Studien zu modellieren. Dabei verwenden sie zum Teil die statistischen Modelle, die Gries eingeführt hat.

Wir hoffen, dass die drei Artikel dieses Themenschwerpunkts zeigen, wie Modellierung die Möglichkeiten von experimenteller Arbeit in der Linguistik ergänzen kann. 


\section{Referenzen}

Ford, Marilyn \& Bresnan, Joan (erscheint) Studying syntactic variation using convergent evidence from psycholinguistics and usage. In: Krug, Manfred \& Schlueter, Julia (eds) Research Methods in Language Variation and Change. Cambridge University Press, Cambridge. Online unter http://www.stanford.edu/ bresnan/ford.bresnan.2010.doc

Gilquin, Gaëtanelle \& Gries, Stefan Th. (2009) Corpora and experimental methods: a state-of-theart review. In: Corpus Linguistics and Linguistic Theory 5(1), 1-26.

Labov, William (2001) Principles of Linguistic Change. Social Factors. Blackwell, Oxford.

Labov, William (2008) Quantitative Analysis of Linguistic Variation. In: Ammon, Ulrich; Dittmar, Norbert; Mattheier, Klaus \& Trudgill, Peter (eds) Sociolinguistics/Soziolinguisik. Vol 1. de Gruyter, Berlin, 6-21.

Manning, Christopher (2003) Probabilistic Syntax. In: Bod, Rens; Hay, Jennifer \& Jannedy, Stefanie (Hrsg.) Probabilistic Linguistics, MIT Press, Cambridge, MA, 289-341.

Wulff, Stefanie \& Gries, Stefan Th. (2011) Corpus-driven methods for assessing accuracy in learner production. In: Robinson, Peter (Hrsg.) Second language task complexity: researching the Cognition Hypothesis of language learning and performance. John Benjamins, Amsterdam, 61-87. 